\title{
Mycoplasma pneumoniae Induced the Expression of Substance $P$ and Its Receptor NK-1 in Human Airway Epithelial Cells
}

\author{
Yujun Zhang, ${ }^{1,2}$ Xiangjun He, ${ }^{1}$ Qi Zhang, ${ }^{1}$ Yulan Liu, ${ }^{1}$ and Yujing Liu ${ }^{1}$
}

This article has been withdrawn as the work was published without the knowledge or approval of the individual who supervised, designed and helped to execute this work.

\footnotetext{
${ }^{1}$ The Central Laboratory, Peking University People's Hospital, Beijing, People's Republic of China

${ }^{2}$ To whom correspondence should be addressed at The Central Laboratory, Peking University People's Hospital, Beijing, People's Republic of China. E-mail: zhangyj@bjmu.edu.cn
} 